\title{
Modelling K shell spectra from short pulse heated buried
}

\section{microdot targets.}

\author{
D J Hoarty ${ }^{1, \text { a) }}$, N Sircombe ${ }^{1}$, P Beiersdorfer ${ }^{2}$, C R D Brown ${ }^{1}$, M P Hill ${ }^{1}$, L M R \\ Hobbs $^{1}$, S F James ${ }^{1}$, J Morton ${ }^{1}$, E Hill ${ }^{3}$, M Jeffery ${ }^{1}, \mathrm{~J}$ W O Harris ${ }^{1}, \mathrm{R}_{\text {Shepherd }}^{2}$, E \\ Marley², E Magee $^{2}$, J Emig ${ }^{2}$, J Nilsen², H K Chung ${ }^{4}$, R W Lee ${ }^{5}$, S J Rose ${ }^{3}$ \\ ${ }^{1} A W E$ plc, Reading, RG74PR, UK. \\ ${ }^{2}$ Lawrence Livermore National Laboratory, Livermore, CA 94550, USA. \\ ${ }^{3}$ Plasma Physics Group, Blackett Laboratory, Imperial College London, London, SW7 2AZ, UK. \\ ${ }^{4}$ NuclearData Section, Divisional of Physical and Chemical Sciences, International Atomic Energy Agency, P O Box \\ 100, A-1400, Vienna, Austria. \\ ${ }^{5}$ Institute for Material Dynamics at Extreme Conditions, University of California, Berkeley, California 94720, USA \\ a) Corresponding author: David.Hoarty@awe.co.uk
}

\begin{abstract}
K shell X-ray emission measurements have been used to diagnose plasma conditions in short-pulse heated buried microdot targets on the Orion high power laser. These experiments have been used to validate simulations of short pulse laser-solid interaction that combine hybrid PIC modelling of the laser absorption with radiationhydrodynamics simulations including an electron transport model. Comparison of these simulations with streaked K shell spectroscopy show the importance of including radial gradients in fitting the spectra. An example is presented of the
\end{abstract}


emission of sulphur from a $50 \mu \mathrm{m}$ diameter microdot sample buried in a plastic foil. Previously agreement between simulation and experiment was obtained only by treating the absorbed energy, electron temperature and beam divergence as fitting parameters. The good agreement obtained in this work used the measured laser energy and laser pulse length and calculated the laser-solid target interaction from first principles.

PACS numbers: $52.50 \mathrm{Jm}, 52.25 \mathrm{Jm}, 52.25 \mathrm{Os}, 52.65 \mathrm{Rr}$

Keywords:

Plasma heating with laser beams

Dense plasma spectroscopy

\section{INTRODUCTION}

Experiments to study dense, high temperature, plasma properties have been conducted over many years using short-pulse high-contrast sub-picosecond laser pulses to heat sample layers buried in low Z tamper foils [1-7]. Though these measurements of dense plasma properties such as ionization potential depression [8,9] and linewidths $[4,10]$ have yielded useful data, the laser-solid target interaction is still not fully understood. To address this there has been an effort to improve the computational models of the laser-target interaction by simultaneously using both the experimental data and the simulation predictions.

Experiments on the UK HELEN laser at AWE showed the importance of the density gradient encountered by the peak intensity short pulse and the effect of pre-pulse irradiation in decoupling the laser energy from the heating of the bulk of the solid target [11]. The modelling of these experiments treated the fraction of the laser energy absorbed into electrons, the mean energy per particle (or the hot electron temperature) and forward directed electron beam divergence as free parameters. These values were altered so that the predicted time history of the plasma conditions matched those of $\mathrm{K}$-shell emission measurements inferred by matching spectra from simulation and experiment. Building on this work, experiments at AWE on the Orion laser [12], used 0.7ps, 100J laser pulses in second harmonic to confirm the HELEN results at higher energy. This paper presents the progress made in calculations of the solid-laser interaction and the heating of buried layer targets from first principles by comparison with sulphur K-shell emission experiments. 
Previous attempts to model the laser-solid interaction problem from first principles using hybrid-PIC models coupled to radiation-hydrodynamics modeling overestimated the degree of heating from first harmonic light, underestimated that produced by second harmonic, and failed to model the effect of the pre-pulse and associated density gradient on the heating of solid targets. These models have been refined by comparison with the results from buried layer experiments [13] and have in turn helped to establish the effect of radial gradients in the interpretation of emitted spectra. In recent work agreement with experimental electron temperatures, pulse durations and temperature profiles has been obtained with the AWE codes using the experimentally measured laser energy and laser pulse duration. The fraction of laser energy absorbed, the mean energy per particle in the electron distribution and the electron beam divergence are no longer fitting parameters but are calculated from first principles.

\section{EXPERIMENTAL METHOD}

On the Orion laser system one of the short pulse laser beams can be converted to second harmonic $(2 \omega, 0.53 \mu \mathrm{m})$ but this can only be done at sub-aperture because the type $1 \mathrm{KDP}$ conversion crystal has to be thin enough ( 2mm maximum) to prevent back-conversion of the $0.7 \mathrm{ps}$ pulse. The diameter of the beam that can be converted is therefore limited by the structural strength of the conversion crystal. In this work the beam diameter was reduced to $30 \mathrm{~cm}$ from the $60 \mathrm{~cm}$ full beam diameter because this is the diameter of the KDP crystal that can be safely handled without breaking. The reduced beam diameter, along with losses due to the conversion, limited the second harmonic energy to $100 \mathrm{~J}$ for the experiments reported here. However, recently an option has been implemented to split the beam and pass it through two square KDP crystals stacked vertically. This allows the conversion of a larger portion of the beam and the energy in second harmonic to be increased to over 200J. Converting to second harmonic, and subsequent reflection from four mirrors to reject any residual unconverted $1.06 \mu \mathrm{m}$ light, reduces pre-pulse levels by many orders of magnitude and sharpens up the leading edge of the pulse which results in more efficient heating of the sample foil $[9,11]$. The targets used in the experiments described here are microdots encased in parylene $\mathrm{N}$ type plastic foils $\left(\mathrm{C}_{8} \mathrm{H}_{8}\right)$. A schematic of the sample is shown in figure 1. The microdot sample is typically $50 \mu \mathrm{m}$ in diameter and buried in parylene $\mathrm{N}$ which, on the front side irradiated with the laser, is $4 \mu \mathrm{m}$ thick and $3 \mu \mathrm{m}$ on the rear. The foil is positioned so that the laser passes through the hole in the $80 \mu \mathrm{m}$ thick plastic washer to irradiate the 
sample foil. The hole is $1 \mathrm{~mm}$ in diameter and the outer washer diameter is typically $2 \mathrm{~mm}$. The laser beam is defocused to the diameter of the microdot or slightly larger. The spot size is limited by the irradiance required to heat the volume of plastic substrates and the sample to the required high temperature.

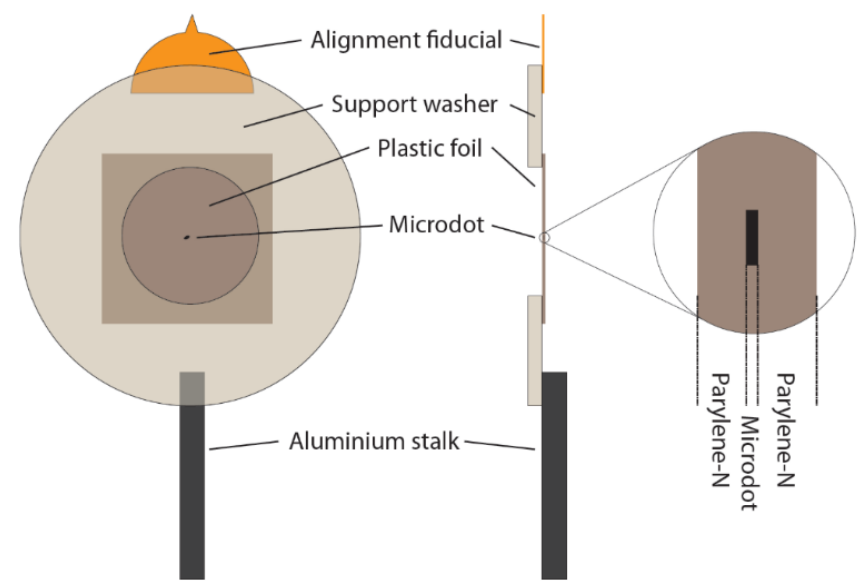

FIGURE 1. (Color online) A schematic of buried microdot targets used at Orion. The microdot diameter is $50 \mu \mathrm{m}$.

The thickness of the parylene $\mathrm{N}$ coatings were chosen based on radiation-hydrodynamics simulations and trials where the duration of the emitted X-ray pulse was measured with an X-ray streak camera with picosecond temporal resolution. The plastic is selected to be thick enough that the rarefaction wave from the outer target surface does not reach the buried microdot until after the duration of the emitted x-ray pulse from the sample. This ensures that the sample stays at high density throughout the spectral measurement. The plastic must also be thin enough so that the laser energy is sufficient to heat the plastic overcoat and sample to high temperature and to ensure that the sample is heated as uniformly as possible over its entire diameter. In the experiments described, the laser irradiance is typically $5 \times 10^{18} \mathrm{~W} / \mathrm{cm}^{2}$. The microdot sample was a sulphide containing an areal density of sulphur of $24 \mu \mathrm{g} / \mathrm{cm}^{2}$ on average. Each microdot was characterized using Rutherford backscattering for thickness, composition and areal density [14] and also white light interferometry for thickness. Further cross checks were performed on witness slides using energy dispersive and wavelength dispersive spectroscopy to check sample composition. A variation of up to $40 \%$ was observed from the characterization which resulted in some sample rejection so that shots in a sequence would use almost identical samples. In addition a significant degree of oxidation was observed, typically around $30 \%$. This was shown to be through the whole sample thickness, the RBS technique allowing sample assay 
layer by layer through the sample by tuning the He ion beam energy. These data, along with the thickness measurements, showed a reduction of a factor of two in the sample density compared to the nominal value at solid.

The sulphur $\mathrm{K}$ shell emission from the heated microdot was measured using an Axis-photonique $\mathrm{x}$-ray streak camera [15] with picosecond resolution coupled to an x-ray crystal spectrometer using a convex cylindrically bent CsAP crystal. The streak camera used a caesium iodide photocathode. The spectra were also recorded timeintegrated using crystal spectrometers recording onto BAS-TR Fuji image plate [16]. The emitting area of the microdot was measured with two instruments on two lines of sight. These were an x-ray pinhole camera with a $5 \mu \mathrm{m}$ diameter pinhole recording onto BAS-TR image plate with eight times magnification viewing $50^{\circ}$ to the target normal and a Kirkpatrick-Baez microscope [17] also recording onto TR image plate with a magnification of ten times viewing $21^{\circ}$ to the target normal. These measurements checked the uniformity of the microdot heating and monitored any structure that may have been imprinted by laser beam spatial structure.

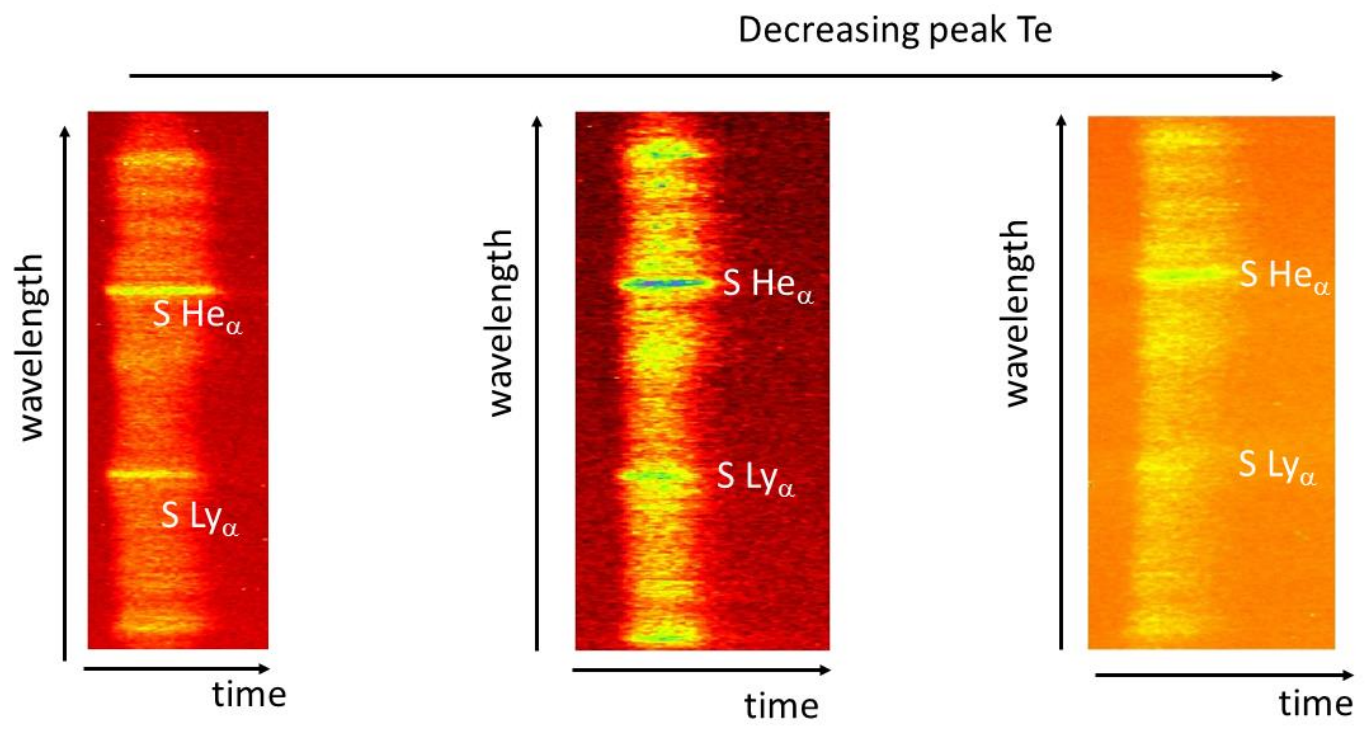

FIGURE 2. (Color online) Streaked sulphur spectra from microdots from three different laser shots over a range of peak electron temperatures varying over $400 \mathrm{eV}$ from left to right (peak Te $1600,1400,1200 \mathrm{eV}$ respectively)

The streaked spectral range was limited to the line transitions from the $1 \mathrm{~s} 2 \mathrm{p}-1 \mathrm{~s}^{2}$ and $2 \mathrm{p}-1 \mathrm{~s}$ lines of He-like and H-like sulphur and the ratio of these transitions was used to infer the time dependent electron temperature during the 
x-ray pulse. The atomic kinetics and spectral synthesis code FLYCHK [18] was used to compare synthetic spectra to the lineouts of the streak spectra to establish the temperature time history. Figure 2 shows the streaked sulphur results from the x-ray streak camera for different incidence irradiance resulting in different peak electron temperatures.

In addition to temporal measurements a slit imaging crystal spectrometer was fielded to record spatially resolved spectra across the focal spot. Results from the microdot targets ranging from 50-100 $\mu \mathrm{m}$ micron diameter showed the temperature across the laser spot diameter and the rapid fall off at the edges of the spot [19]. The data also showed a 20-30 $\mu \mathrm{m}$ diameter emitting area even when the beam was fired at best focus, which was measured at an equivalent plane focal spot monitor as $10-15 \mu \mathrm{m}$, showing that there was significant enlargement of the $\mathrm{x}$-ray emitting area compared to the laser spot at the highest irradiances. However, for a defocused beam, $50 \mu \mathrm{m} \mathrm{FWHM}$ spot, the x-ray emitting area corresponded closely to the laser spot. This effect was replicated in the simulations with the increase in the x-ray emitting area diameter compared to the focused laser spot attributed to electrons refluxing laterally between the fields produced at the sample-tamper boundaries by fast electron transport during the laser pulse.

\section{DATA ANALYSIS AND MODELLING}

Previous modelling of buried layer targets used a Gaussian time-dependent energy deposition over the duration of the laser pulse input to a radiation-hydrodynamics model to produce a time history of the sample conditions. The energy deposited was treated as a free parameter and iterated with the atomic kinetics calculations until best fit was obtained between the measured spectrum and the synthetic spectrum predicted by the atomic kinetics model using the calculated time history [9]. The main problem with this energy deposition approach is that it does not account for radial gradients. A more sophisticated treatment has been developed over a number of years that uses a treatment of the laser solid-target interaction from first principles using the EPOCH [20] PIC code coupled to a radiation-hydrodynamics model, CORVUS running an in-line electron transport model THOR [13, 21]. This approach has been refined by improving the linkages between hydrodynamics and the THOR electron transport 
model within CORVUS; improving the materials properties used in the THOR/CORVUS simulations and improvements to the linkages between EPOCH and the THOR/CORVUS model. Improvements in the measurement of the pre-pulse at the fundamental wavelength and the on target laser energy, through reflectivity measurements of the final optics, have also led to the large improvement in the agreement between simulation and experimental observations. The linkage between codes is illustrated in figure 3 .

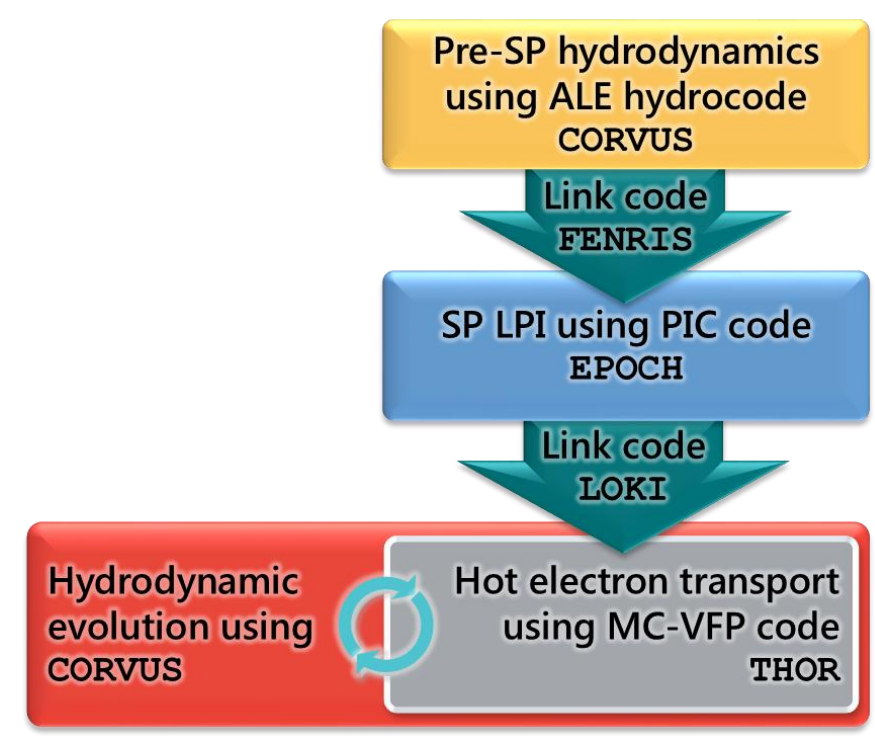

FIGURE 3. (Color online) Flow diagram for the calculation of sample conditions from first principles. Typically 2D Cartesian planar geometry is used throughout although 3D Cartesian PIC coupled to 2D cylindrical hydrodynamics is an option.

The effect of a laser pre-pulse can be calculated using the radiation-hydrodynamics code CORVUS and used as input to EPOCH to take the density gradient into account. With frequency conversion the $2 \omega$ pre-pulse is so low the pulse contrast is below the detection threshold of the Orion instrumentation. The $2 \omega$ pre-pulse is included in the modelling as the square of the pulse contrast at the fundamental frequency. The EPOCH model calculates the electron source from the laser source, modelling the laser plasma interaction directly, which is then linked to the radiation-hydrodynamics via the LOKI link code. The electrons are then transported using the THOR Monte-Carlo, Vlasov-Fokker Planck code which sub-cycles in the radiation-hydrodynamics code CORVUS [21]. 
The material properties are calculated from an in-line non-LTE XSN type screened-hydrogenic averageatom model Zeus and for lower temperatures tabulated EoS and opacity tables. The fundamental assumption in the electron heating is that the forward directed current transported through the target is balanced by a thermal return current from the plasma background due to the field produced by the displaced electrons. The bulk of the heating in the layer over the timescale of the diagnostic is due to the Ohmic heating of the plasma by this return current [11] with the resistivity calculated from a capped Spitzer model [21]. In this modelling, the energy and pulse length used in the calculations of the temperature and density distributions, to give a best fit between measured and simulated spectra, were the measured on shot values. The energy uncertainty on the laser energy measurement of $+/-5 \%$. The uncertainty on the pulse length is $+/-20 \%$.
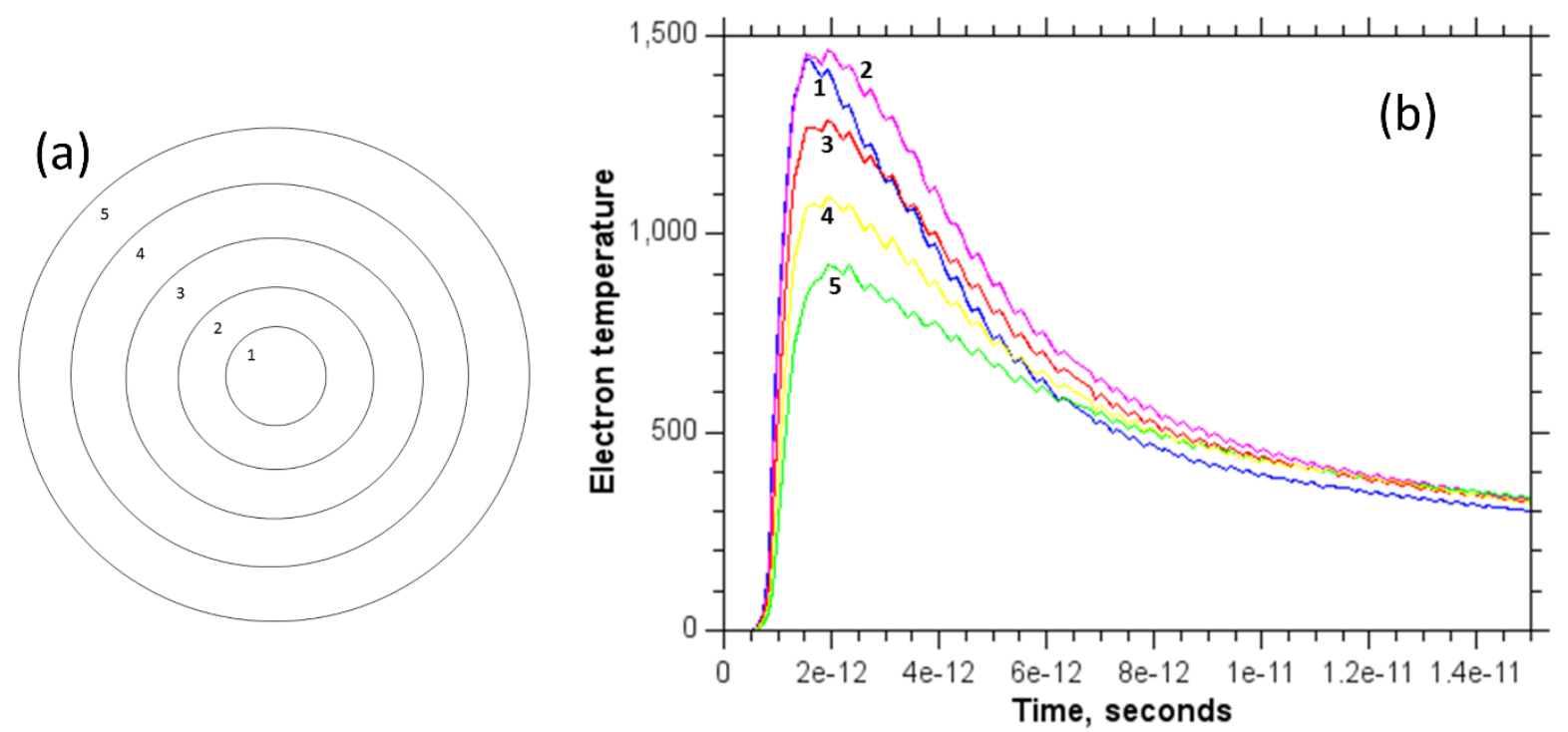

FIGURE 4. (Color online) A schematic showing the division of the microdot into annuli labelled 1-5 in panel (a). The right panel (b) shows curves of the electron temperature averaged over the area of the each annulus plotted following the same labelling.

To generate the synthetic spectra from predictions of the EPOCH/THOR/CORVUS calculations, the microdot radius was divided every $5 \mu \mathrm{m}$ and the sample thickness was divided into three zones; front, back and centre and the mean values taken from each of these zones for each annulus. The contribution to the streak camera signal was taken as that of the three transverse zones from each annulus weighted by its area at each time taken in experimental 
lineout. The temperature as a function of time for the different annuli are plotted in figure 4 . The atomic kinetics models used were FLYCHK and ALICE [22]. Most of the calculations were carried out using FLYCHK with some results repeated using ALICE to check the effect of opacity on the sulphur line ratios. The ALICE code calculates the effect of opacity by solving the equation of radiation transfer which is a more rigorous treatment than the escape factor method used in FLYCHK. However, for the cases considered here, the two codes were in close agreement on the inferred temperatures from the line ratios.

The fall-off in the electron temperature of the outer annuli reflects the Gaussian spatial intensity distribution of the $50 \mu \mathrm{m}$ FWHM spot. The largest radial gradient is at the time of peak electron temperature just after the end of the laser pulse. Although the inner $30 \mu \mathrm{m}$ diameter of the spot shows only a $200 \mathrm{eV}$ variation falling to about $100 \mathrm{eV}$ at 4 ps and less than $50 \mathrm{eV}$ at times after this. By $7 \mathrm{ps}$ the temperatures over the whole spot show about a $100 \mathrm{eV}$ variation. Due to the radiative loss from the microdot exceeding that of the low density tamper the outermost zone is at similar temperature to the centre by $8-9$ ps onwards due to thermal conduction from the surrounding low $\mathrm{Z}$ tamper. The lineouts at each time in the streak camera measurements are the superposition of the transverse and radial gradients in the microdot averaged over the lineout time interval that was typically one to two picoseconds. Changing the laser irradiance, or reducing the overcoat of plastic between the microdot and the laser, has the effect of changing the peak electron temperature and radial gradient in the first few picoseconds of emission. At later times, i.e., 5ps onwards, because higher temperature results in a greater emitted intensity and more rapid cooling, the temperature is similar in high or lower irradiance cases over the range of $2 \times 10^{18}-2 \times 10^{19} \mathrm{~W} / \mathrm{cm}$ investigated in these experiments. The temporal gradient could therefore be reduced by reducing the peak temperature, either by burying the microdot in a thicker overcoat than the $4 \mu \mathrm{m}$ case shown here, or reducing the irradiance. Using a smaller microdot of $30 \mu \mathrm{m}$ diameter would also reduce the radial gradient, as shown from the inner three zones on the plot in figure 4b. Producing microdots with a top hat profile on the microdot thickness, however, has proved difficult, with measurements showing a domed profile for diameters less than $50 \mu \mathrm{m}$. Alternatively, increasing the size of the focal spot, so that the fall off seen in the outer zones of the microdot occurs in the plastic tamper surrounding the microdot, would ensure a much more uniform microdot temperature. 
Note that the rapid increase in the temperature in the initial picosecond or so of the curves in figure $4 \mathrm{~b}$ is not recorded on the streak camera because of a combination of the temporal resolution limit and the detection threshold. The streak records the K-shell emission from the electron temperature peak onwards. Lineouts through the streak data are an integration of at least $1 \mathrm{ps}$ of the emitted X-ray pulse due to the need to average to improve the signal-tonoise ratio. Figure 5 shows the streaked sulphur data from a microdot target at the conditions shown in figure 4 .
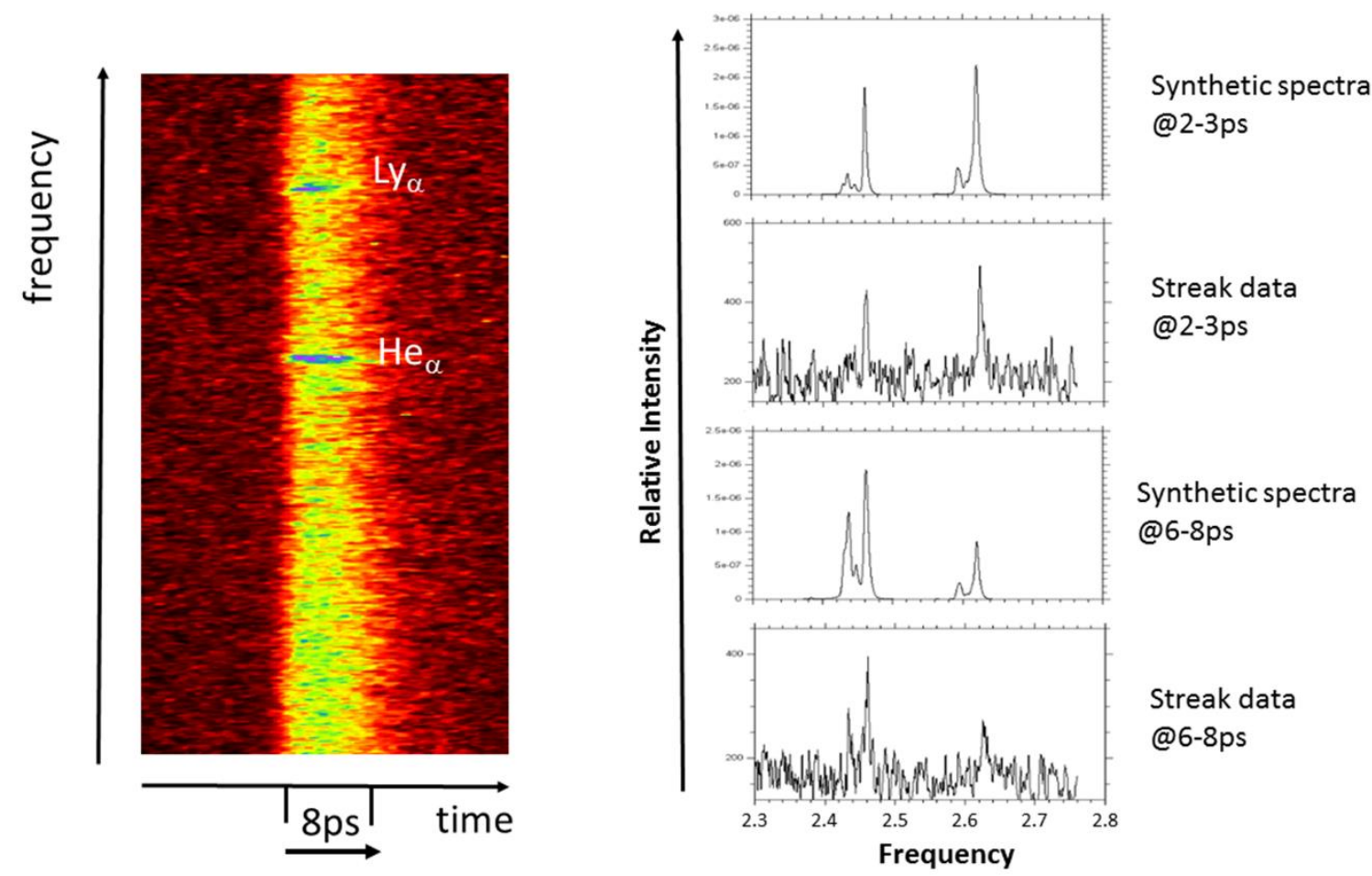

FIGURE 5. (Color online) A measured streaked sulphur emission spectrum and synthetic spectra generated at the conditions shown in figure $4 \mathrm{~b}$ showing the $\mathrm{K}$ shell emission from He-like and Ly-like sulphur lines and satellites. The panels on the right show lineouts at different times compared to FLYCHK simulations which are the integration of the spectra predicted accounting for the gradients shown in figure $4 \mathrm{~b}$. The streaked lineouts are integrated over 1 or 2 ps to reduce noise.

The synthetic emission spectra obtained from each transverse zone and annulus is modelled using the EPOCH/THOR/CORVUS conditions input to FLYCHK simulations and an area weighted emission spectrum produced taking the gradients into account for each lineout. These synthetic spectra are then compared with the 
lineouts from the streak data for the same times. The panel on the right of figure 5 shows an example of results of the modelling compared to lineouts near the beginning and the end of the streaked $\mathrm{x}$-ray pulse. Good agreement is obtained between simulation and measurement in the emitted spectral line ratios as a function of time and in the duration of the emission recorded on the camera before the signal intensity falls and is indistinguishable from the streak camera noise.

\section{SUMMARY/CONCLUSION}

Modelling of $\mathrm{K}$ shell emission spectra to obtain plasma conditions in short pulse heated experiments has been validated by comparison of streaked sulphur K-shell line measurements and simulated spectra generated from calculation combining hybrid PIC, electron transport and radiation-hydrodynamics. Good agreement has been obtained without recourse to altering the input parameters of deposited energy, electron beam temperature or beam divergence. The values used for the energy and pulse-length of the laser were those measured on the experiment and the laser-solid-target interaction was calculated from first principles by the particle-in-cell code EPOCH. The electron distribution obtained was then used as input to an electron transport model running in-line with the AWE radiation-hydrodynamics code CORVUS. The simulations show the effect of radial and transverse gradients, as well as temporal gradients, and show that these must be taken into account in a two dimensional model to match the streak camera measurements of the time dependent K-shell spectra. It is shown that radial gradients can be greatly reduced by using microdots smaller that the laser spot. For future experiments on Orion it will be possible to increase the size of the focal spot, so that the fall off seen in the outer zones of the microdot occurs in the plastic tamper surrounding the microdot, to ensure a much more uniform microdot temperature as discussed in section 3. The increase in $2 \omega$ energy now available due to the increased beam aperture, as described in section 2 , will allow the focal spot to be increased while keeping the irradiance the same as the example case shown in figure 4.

The synthetic spectra in the study were generated from the simulated plasma conditions using the atomic kinetics models FLYCHK and ALICE. The two approaches were in good agreement for the sulphur cases modelled. Comparison of the solution of the full radiation transfer equation using ALICE and the escape factor treatment of FLYCHK, suggests that the escape factor treatment in FLYCHK was a good approximation in this case. The results 
will be used to refine the experimental designs for future high temperature and density opacity experiments using buried microdot samples. It is worth noting that good agreement between time integrated spectra and one dimensional energy deposition simulations, as described at the beginning of section 3, can be obtained even when the agreement with streak measurements of the K shell time-history is poor. Streaked spectra are crucial to fix the sample conditions and properly account for gradients. Ideally, several low Z, K shell emission spectra from the target should be used to obtain the sample conditions. Typically, Orion experiments use a mix of time-resolved and time-integrated data from low $\mathrm{Z}$ materials such as sulphur, silicon, aluminium, chlorine, potassium and scandium. Each material has an optimum temperature sensitivity range so that as lower $\mathrm{Z}$ materials, such as aluminium and silicon, become insensitive to temperature due to ionization to predominantly hydrogen-like ions, K-shell emission from higher $\mathrm{Z}$ materials, such as sulphur or scandium, will still have a significant helium-like population to allow accurate $\mathrm{He} / \mathrm{H}$ like line ratio comparisons. In the example shown the target also had a layer of scandium adjacent to the sulphur. Although the scandium emission was not time resolved it was modelled and was able to provide an additional constraint on the plasma conditions.

While results presented here are from two dimensional simulations, preliminary simulations using the EPOCH/THOR/CORVUS in three dimensions suggest that the fall off in the electron temperature at the edges of the laser spot and also transversely through the target is more rapid than the two dimensional case due to additional divergence of the electron beam. The contribution to the measured spectra from the outer edge of the microdot is predicted to be small compared to that from the centre in two and three dimensional calculations.

\section{FUTURE MODEL DEVELOPMENT}

As well as extension to three dimensions, additional physics is being considered for the models. At present the non-local transport is confined to the electrons accelerated by the laser field and electron transport in the background electrons is calculated using flux limited diffusion. Investigation of the choice of flux limiter has shown that it does not change the inferred spectra though the transverse gradient in the sample does change. Another effect under consideration is ponderomotive pressure. The effect of the ponderomotive pressure is calculated in EPOCH so that hole-boring and steepening of the critical density surface by this effect is taken into account in the calculation of 
the laser absorption. This pressure is not mapped across to the THOR/CORVUS calculation and so is not accounted for in the subsequent radiation-hydrodynamics. At the intensities considered in this work it is not expected to be significant compared to the thermal pressure from the heated plasma, but ponderomotive pressure may affect the hydrodynamics for higher irradiances. More generally, in spite of the good agreement obtained with the THOR model of electron transport shown in this work, there remain uncertainties in the physics of the laser-solid interaction. A parallel approach by Sherlock [23] combining two dimensional hybrid PIC modelling with electron transport and radiation-hydrodynamics, has shown heating indistinguishable from the EPOCH/THOR/CORVUS modelling despite the dominant energy transfer mechanism from the laser to the solid target not being due to the return current but rather the damping of electron plasma waves in the wake of electron bunches accelerated into the target by each cycle of the laser field. Further work is still needed to understand the microphysics of the laser-solid interaction.

\section{AKNOWLEDGEMENTS}

The authors would like to acknowledge the help and dedication of the staff of the Orion laser and AWE target fabrication. This paper is published under (C British Crown Owned Copyright 2017/AWE.

\section{REFERENCES}

1. K Nazir et al., Appl. Phys. Lett. 69, 3686 (1996)

2. B. K. F. Young et al., Phys. Rev. E, 58, 4929 (1998)

3. A. Saemann et al., Phys. Rev. Lett. 82, 4843 (1999)

4. K Eidmann et al., J. Quant. Spectrosc. Radiat. Transfer 81, 133 (2003)

5. R. G. Evans et al., Appl. Phys. Lett. 86, 191505 (2005)

6. D. J. Hoarty et al., High Energy Density Phys. 3, 325 (2007)

7. V. Dervieux et al., High Energy Density Phys. 16, 12 (2015)

8. D. J Hoarty et al., Phys. Rev. Lett. 110, 265003 (2013)

9. D. J. Hoarty et al., High Energy Density Phys, 9, 661 (2013)

10. P. Beiersdorfer et al., Rev. Sci. Instrum. 87, 063501 (2016)

11. C. R. D. Brown et al., Phys. Rev. Lett. 106, 185003 (2011) 
12. N. Hopps et al., Plasma Phys. Control. Fusion, 57, 064002 (2015)

13. N. J. Sircombe et al., J. Phys. Conf. Series, 717, 012081 (2016)

14. C. Jeynes et al., Analytical Chemistry, 84, 6061 (2012)

15. C. Y. Coté, Axis Photonique Inc. Varennes, QC, Canada

16. N. Izumi et al., Rev. Sci. Instrum. 77, 10E325 (2006)

17. P. Kirkpatrick and A. V. Baez, J. Opt. Sci. Am. 38, 766 (1948)

18. H. K. Chung et al., High Energy Density Phys, 1, 3 (2005)

19. D. J Hoarty et al., Atomic Processes in Plasmas, J. Conf. Proc. confproc@aip.org in Press

20. T. D. Arber et al., Plasma Phys. Control. Fusion, 57, 113001 (2015)

21. N. J. Sircombe, S. J. Hughes and M. G. Ramsey, New J. Phys. 15, 025025 (2013)

22. E. G. Hill and S. J. Rose, High Energy Density Phys. 8, 307 (2012)

23. M. Sherlock et al., Phys. Rev. Lett. 113, 255001 (2014) 